Revista

\title{
Multi-Ensayos
}

Vol. 6, núm. 11

ISSN: 2412-3285

https://multiensayos.unan.edu.ni

DOI: https://doi.org/10.5377/multiensayos.v6i11.9282

\section{Rúbrica, un camino para evaluar objetivamente el aprendizaje en el aula virtual}

\section{Rubric, a path to objectively assess learning in the virtual classroom}

\author{
Gregoria Casco Casco ${ }^{1}$ \\ Alba Deyanira Calderón²
}

Recibido: 25 de octubre de 2019. Aceptado: 14 enero de 2020

\section{RESUMEN}

La rúbrica es un instrumento de evaluación cuya finalidad es compartir criterios o indicadores, que permitan evaluar de manera objetiva y crítica el aprendizaje adquirido por los estudiantes, las habilidades desarrolladas de acuerdo a los objetivos. Por lo tanto, este instrumento se hace necesario cuando se facilitan asignaturas de manera virtual, porque a través de ella, el estudiantado es capaz de valorar sus logros y dificultades. De la misma forma, el docente retroalimenta a partir de las debilidades encontradas con el fin de la mejora y reincorporación de sugerencias. Es por ello, que es necesario reflexionar desde el punto pedagógico y didáctico sobre las características, estructura, diseño y utilización de la rúbrica para la evaluación de los aprendizajes del estudiantado; considerando que la rúbrica permite la participación e interacción de los diferentes actores del proceso de enseñanza - aprendizaje.

Palabras claves: rúbrica; evaluación; aprendizaje.

\section{ABSTRACT}

A rubric is an evaluation tool whose purpose is to share criteria or indicators, which allow evaluating objectively and critically students' learning, as well as the skills developed according to the objectives. Therefore, this instrument is necessary when teaching online because students can assess their achievements and difficulties. Likewise, the teacher can provide feedback to strengthen the weaknesses found to overcome them. It is, therefore, necessary to reflect from the pedagogical and didactic point of view on the characteristics, structure, design, and use of the rubric for the evaluation of student apprenticeships, considering that the rubric allows the participation and interaction of the different actors of the teaching-learning process.

Keywords: rubric; assessment; learning.

1 Docente UNAN-Managua/FAREM-Estelí. Correo electrónico: gregoriacasco@yahoo.es.

2 Docente UNAN-Managua/FAREM-Estelí. Correo electrónico: albacalderon@yahoo.es.

(c) 2020 Revista Multi-Ensayos. 


\section{INTRODUCCIÓN}

La rúbrica es un instrumento que fomenta la credibilidad de la evaluación, vista desde los diferentes actores que participan en el proceso de aprendizaje. Es por ello, que el propósito de este ensayo es analizar la importancia de la aplicación de la rúbrica como forma de evaluación de los conocimientos, habilidades y capacidades adquiridas, y desarrolladas en las diferentes asignaturas virtuales.

El proceso de enseñanza - aprendizaje permite las interacciones entre los diferentes actores del proceso educativo. Si este se facilita de manera presencial permite el intercambio entre los protagonistas y observar la participación de cada uno de ellos; sin embargo, cuando este proceso se desarrolla de manera virtual se necesita de la búsqueda y aplicación de un instrumento, que conlleve a evaluar los conocimientos, habilidades adquiridas de manera objetiva y es aquí la importancia de la rúbrica para evaluar.

\section{DESARROLLO}

Tradicionalmente se ha utilizado diferentes formas de evaluación, entre ellas una de las más comunes es la prueba escrita. Sin embargo, actualmente en la plataforma Moodle, donde se facilita algunas asignaturas, como es el caso de Técnicas de Lectura, Redacción y Ortografía en la Universidad Nacional Autónoma de Nicaragua (UNAN-Managua) se está promoviendo el uso de la rúbrica para la evaluación de las actividades de aprendizajes. Esta contribuye a valorar los procedimientos aplicados para la gestión del conocimiento y resolución de los ejercicios propuestos de acuerdo a las competencias y contenidos que deben desarrollar los estudiantes en cada una de las unidades.

En todo proceso de enseñanza-aprendizaje requiere de la aplicación de estrategias metodológicas y estas a su vez, de un instrumento de evaluación. Por ejemplo, para evaluar una exposición es necesario la elaboración de una rúbrica en la cual, se define los criterios a evaluar, lo que permite que el evaluado conozca anticipadamente; ¿Qué se evalúa?

Partiendo de lo anterior es necesario reflexionar sobre, ¿qué es una rúbrica? ¿Cuáles son sus características? ¿Qué elementos contiene?

Alsina \& otros (2013, p. 8) expresan que una rúbrica "...es un instrumento cuya principal finalidad es compartir los criterios de realización de las tareas de aprendizaje y de evaluación con los estudiantes y entre el profesorado." También agregan que, "La rúbrica es un potente instrumento para la evaluación de cualquier tipo de tarea, pero hay que destacar especialmente su valor para evaluar tareas auténticas, tareas de la vida real." (p. 10)

Por ende, la rúbrica es un medio que tiene como finalidad certificar tanto de forma cualitativa como cuantitativa el nivel o grado de aprendizaje construido a través de las diferentes actividades o sugerencias de trabajo. Además, conlleva a despertar el interés y el deseo de superación en las dificultades encontradas 
gracias a la oportuna retroalimentación por parte del docente. Por ejemplo: en cada una de las semanas del desarrollo programático de la asignatura Técnicas de Lectura, Redacción y Ortografía facilitada de forma virtual, se presenta una rúbrica para evaluar las actividades de aprendizaje programadas, tomando en cuenta los objetivos de acuerdo al contenido, que el estudiante debe alcanzar al finalizar la misma.

Por consiguiente, el docente como sujeto activo en la evaluación del aprendizaje debe contemplar en la elaboración de la rúbrica una serie de elementos que la caracterizan. De acuerdo a Valverde \& Ciudad (2014, p. 55) las características principales de una rúbrica son: "Instrumento vinculado a la evaluación auténtica. Fiabilidad y la validez de los hallazgos." Esto facilita que el estudiante sea consciente de su propio aprendizaje, detecte sus dificultades y corrija errores tomando en cuenta las sugerencias del docente con fin hacia la mejora de la calidad de su aprendizaje.

En cambio (Popham, 1997) citado por Valverde \& Ciudad (2014, p. 49) considera que las rúbricas contienen tres características fundamentales: unos criterios de evaluación, una escala de valoración y una estrategia de calificación. Esta consideración permite valorar y calificar el nivel de conocimiento de acuerdo al ritmo de aprendizaje de cada estudiante, y por ende, la calidad de las actividades de aprendizaje asignadas en cada sesión.

A continuación se explica los aspectos que conforma cada una de las características propuestas por Popham.

Criterio de evaluación

-...establece cuáles son los elementos sobre los que va a bas

- ar la evaluación de los aprendizajes, ofrece al estudiante los aspectos esenciales de la tarea que será objeto de evaluación por parte del profesorado.

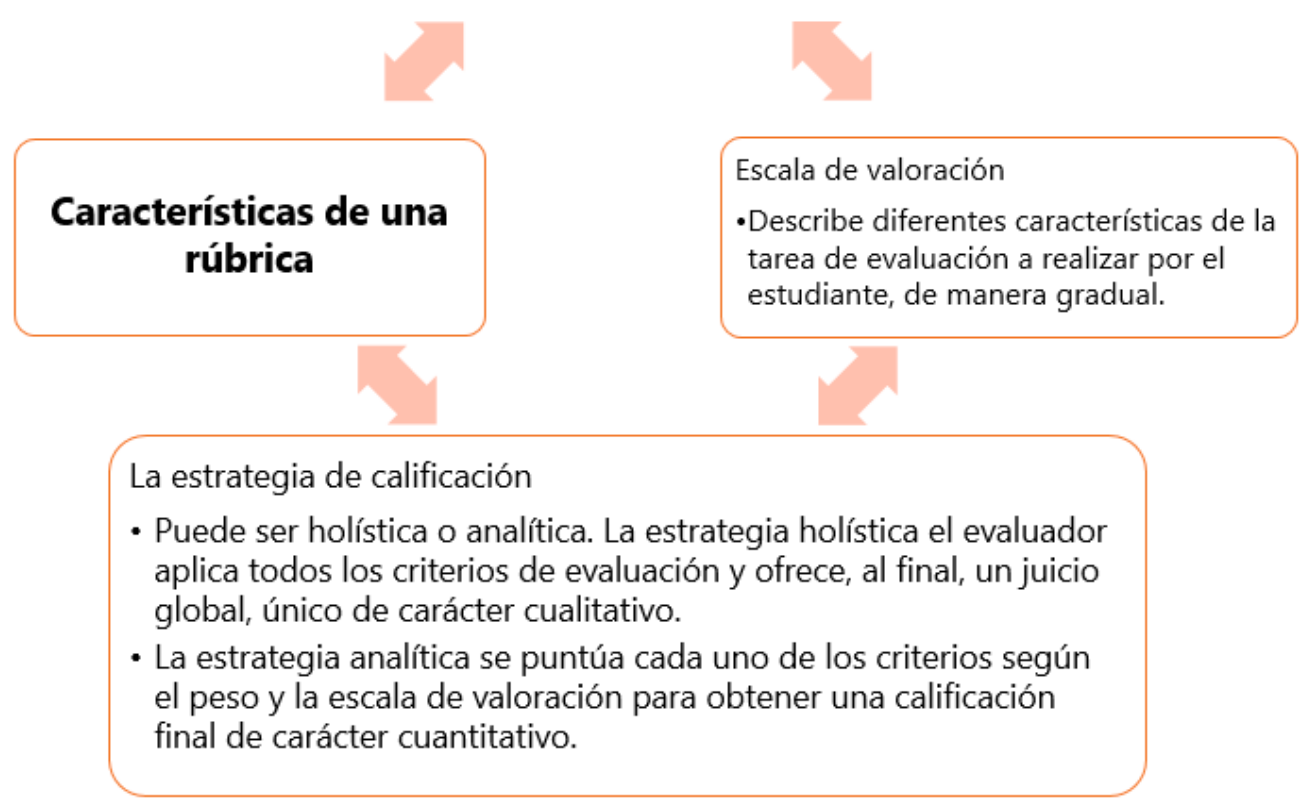

(Popham, 1997) citado por Valverde \& Ciudad (2014, p. 49). 
Un elemento importante, que toda rúbrica debe poseer son los indicadores de evaluación bien definidos, esto en dependencia de los objetivos a evaluar. Para ver reflejadas las tres características antes señaladas, se presenta un ejemplo de rúbrica utilizada en el aula virtual para la evaluación de la redacción de un ensayo argumentativo.

\section{RÚBRICA DE EVALUACIÓN DEL ENSAYO}

La rúbrica que se presenta a continuación especifica los criterios de evaluación que se tomarán en cuenta en la revisión del ensayo de Técnicas de Lectura Redacción y Ortografía. Estos criterios, serán considerados para emitir un juicio sobre aspectos específicos del trabajo final. Su diseño se ha realizado a partir del análisis minucioso de la actividad realizada.

\begin{tabular}{|c|c|c|c|c|c|}
\hline $\begin{array}{l}\text { Criterio de } \\
\text { Evaluación }\end{array}$ & Excelente & Muy Bueno & Bueno & Regular & Deficiente \\
\hline $\begin{array}{l}\text { Presenta la } \\
\text { información } \\
\text { pertinente en la } \\
\text { introducción. }\end{array}$ & $\begin{array}{l}\text { La introducción es un párrafo y } \\
\text { cuenta con los tres elementos: la } \\
\text { presentación del tema, el objetivo } \\
\text { y la tesis. }\end{array}$ & $\begin{array}{l}\text { La introducción es un párrafo y } \\
\text { cuenta: dos elementos } \\
\text { correspondientes a la parte } \\
\text { introductoria. }\end{array}$ & $\begin{array}{l}\text { La introducción es un párrafo } \\
\text { y cuenta: un elemento } \\
\text { correspondiente a la parte } \\
\text { introductoria. }\end{array}$ & $\begin{array}{l}\text { La introducción es párrafo, pero no } \\
\text { cuenta con ninguno de elementos } \\
\text { correspondientes a la parte } \\
\text { introductoria. }\end{array}$ & $\begin{array}{l}\text { No cumplió con } \\
\text { el trabajo. }\end{array}$ \\
\hline $\begin{array}{l}\text { Estructuración de los } \\
\text { argumentos del } \\
\text { escrito conforme al } \\
\text { bosquejo. }\end{array}$ & $\begin{array}{l}\text { Los argumentos presentados en el } \\
\text { escrito final corresponden a la } \\
\text { estructura del bosquejo. }\end{array}$ & $\begin{array}{l}\text { Un argumento presentado en } \\
\text { el escrito final no corresponden } \\
\text { a la estructura del bosquejo. }\end{array}$ & $\begin{array}{l}\text { Dos argumentos presentados } \\
\text { en el escrito final no } \\
\text { corresponden a la estructura } \\
\text { del bosquejo. }\end{array}$ & $\begin{array}{l}\text { Tres argumentos presentados en el } \\
\text { escrito final no corresponden a la } \\
\text { estructura del bosquejo. }\end{array}$ & $\begin{array}{l}\text { No cumplió con } \\
\text { el trabajo. }\end{array}$ \\
\hline $\begin{array}{l}\text { Estructura de los } \\
\text { párrafos }\end{array}$ & $\begin{array}{l}\text { Todos los párrafos tienen una idea } \\
\text { principal y tres ideas secundarias. }\end{array}$ & $\begin{array}{l}\text { Cuatro párrafos tienen una } \\
\text { idea principal y dos ideas } \\
\text { secundarias. }\end{array}$ & $\begin{array}{l}\text { Tres párrafos tienen una idea } \\
\text { principal y dos ideas } \\
\text { secundarias. }\end{array}$ & $\begin{array}{l}\text { De dos a un párrafo tienen la idea } \\
\text { principal y dos ideas secundarias }\end{array}$ & $\begin{array}{l}\text { No cumplió con } \\
\text { el trabajo }\end{array}$ \\
\hline Coherencia & $\begin{array}{l}\text { Las ideas se presentan en orden } \\
\text { lógico, existe progresión temática, } \\
\text { exactitud y pertinencia (las ideas } \\
\text { principales y secundarias están } \\
\text { estrechamente relacionadas) }\end{array}$ & $\begin{array}{l}\text { Las ideas presentan errores de } \\
\text { orden lógico, pero posee } \\
\text { pertinencia, progresión } \\
\text { temática y exactitud en las } \\
\text { ideas. }\end{array}$ & $\begin{array}{l}\text { Algunas ideas no presentan, } \\
\text { pertinencia, orden lógico y } \\
\text { exactitud, pero existe } \\
\text { progresión temática. }\end{array}$ & $\begin{array}{l}\text { Las ideas no presentan orden lógico y } \\
\text { no existe progresión temática. } \\
\text { Falta pertinencia entre las ideas } \\
\text { principales y secundarias. } \\
\text { No plantea exactitud en las ideas. }\end{array}$ & $\begin{array}{l}\text { No cumplió con } \\
\text { el trabajo }\end{array}$ \\
\hline $\begin{array}{l}\text { Presentación del } \\
\text { escrito }\end{array}$ & $\begin{array}{l}\text { El escrito final está conforme a la } \\
\text { estructura del ensayo } \\
\text { argumentativo. }\end{array}$ & $\begin{array}{l}\text { El escrito final no está conforme a la } \\
\text { estructura del ensayo argumentativo }\end{array}$ & $\begin{array}{l}\text { El escrito final está conforme a la } \\
\text { estructura del ensayo pero exister } \\
\text { errores de coherencia e ideas sin } \\
\text { desarrollar }\end{array}$ & $\begin{array}{l}\text { El escrito final no está conforme a la } \\
\text { estructura del ensayo argumentativo. }\end{array}$ & $\begin{array}{l}\text { No cumplió con } \\
\text { el trabajo }\end{array}$ \\
\hline Conclusión & $\begin{array}{l}\text { La conclusión es fuerte y deja al } \\
\text { lector con una idea absolutamente } \\
\text { clara con referente a la situación } \\
\text { del tema abordado. }\end{array}$ & $\begin{array}{l}\text { La conclusión trasmite la } \\
\text { posición del autor, pero no es } \\
\text { convincente. }\end{array}$ & $\begin{array}{l}\text { La conclusión es evidente, } \\
\text { pero no clara la situación del } \\
\text { tema abordado. }\end{array}$ & $\begin{array}{l}\text { La conclusión es solo un párrafo más } \\
\text { del escrito sin idea fundamental que } \\
\text { refleje la situación del tema } \\
\text { abordado. }\end{array}$ & $\begin{array}{l}\text { No cumplió con } \\
\text { el trabajo }\end{array}$ \\
\hline $\begin{array}{l}\text { Ortografía y } \\
\text { redacción }\end{array}$ & $\begin{array}{l}\text { Toda la ortografia y gramática es } \\
\text { correcta }\end{array}$ & $\begin{array}{l}\text { Contiene menos de } 5 \text { errores } \\
\text { de ortografia y redacción }\end{array}$ & $\begin{array}{l}\text { Contiene más de } 10 \text { errores } \\
\text { de ortografia y redacción. }\end{array}$ & $\begin{array}{l}\text { Contiene más de } 15 \text { errores de } \\
\text { ortografia y redacción }\end{array}$ & $\begin{array}{l}\text { No cumplió con } \\
\text { el trabajo }\end{array}$ \\
\hline
\end{tabular}

Con la aplicación de la rúbrica como instrumento de evaluación se logra muchos beneficios tanto para el estudiante como para el docente, entre los que se encuentran:

...ayuda a los profesores a definir la excelencia y el plan de instrucción, alinea los objetivos del currículo y la propuesta de evaluación, contribuye a ser cuidadosos, honestos y consistentes en las calificaciones, reduce el tiempo utilizado para evaluar el trabajo del estudiante, puede promover la consistencia entre las expectativas y los resultados de cursos, departamentos, etc.

En los estudiantes, la rúbrica aclara las expectativas de los profesores, crea un alto nivel de exigencia con una realización de calidad claramente indicada, ayuda en la autoevaluación y la evaluación recíproca y proporciona un feedback orientado que identifica cómo y dónde hay que mejorar (García, 2013, p. 92). 
Lo anterior, demuestra que la utilización de rúbricas en la evaluación de proceso y formativa es funcional, porque promueve la autoevaluación, coevaluación y heteroevaluación de los aprendizajes mediante la integración y participación de los actores. Por consiguiente, es importante que el estudiante sea consciente, crítico, autocrítico y honesto; en otras palabras, que posea valores y principios éticos para lograr de esta manera ser objetivo en la evaluación de su propio aprendizaje. Tal como lo ratifica, Elena (2015) al decir:

El empleo de la rúbrica para el desarrollo de la autorregulación desde la autoevaluación, la coevaluación y heteroevaluación estimula a aprender a aprender. Esto permite valorar a partir de diferentes actores el progreso del aprendizaje construido por el estudiante; tomando en cuenta los criterios y las evidencias de las tareas $o$ actividades de aprendizaje orientadas.

\section{CONCLUSIÓN}

La rúbrica por sus características contribuye a lograr la interacción de forma dinámica, activa y propositiva de los actores principales durante el proceso de evaluación de los aprendizajes. Lo que conlleva a una valoración más objetiva desde las diversas formas de evaluación utilizada en la resolución de actividades aprendizajes propuestas para el logro de las competencias propias de la asignatura de Técnicas de Lectura, Redacción y Ortografía.

Además fomenta en los estudiantes la capacidad de reconocer sus errores y la búsqueda de la mejora continua de sus aprendizajes a través de la aplicación de conocimientos, habilidades y hábito para lograr un aprendizaje autónomo y consciente despertando la curiosidad, y la investigación oportuna. Mientras que al docente valorar el aprendizaje de los estudiantes de manera más objetiva mediante la evaluación de proceso.

Es por eso que la rúbrica es un instrumento que debe ser utilizado para una evaluación creíble vista desde los diferentes actores que participan en el proceso enseñanza-aprendizaje; es decir a través de la rúbrica, no solamente el docente evalúa el aprendizaje, sino que también sus compañeros de clase, pero sobre todo cuando el estudiante es capaz de valorar su propio trabajo.

\section{REFERENCIAS}

Alsina, J., Argila, A., Aróztegui, M., Arroyo, J., Badia, M., \& Carreras, A. (2013). Cuadernos de docencia universitaria. Barcelona: OCTAEDRO, S. L. Recuperado el 25 de Junio de 2019, de http://www. ub.edu/ice/sites/default/files/docs/qdu/26cuaderno.pdf

Elena, C. (2015). La rúbrica como instrumento de evaluación de competencias en educación superior: ¿Uso o abuso? Profesorado, 264-280.

García, M. (2013). La evaluación de competencias de Educación Superior mediante rúbricas: un caso práctico. Revista electrónica interuniversitaria de formación del profesorado, 87-106.

Valverde , J., \& Ciudad, A. (2014). Uso de e-rúbricas para la evaluación de competencias en estudiantes universitaria. Estudio sobre fiabilidad del instrumento. Revista de docencia Universitaria, 49-79. 